\title{
Resultados obstétricos y perinatales asociados a la infección por COVID-19: una revisión de la literatura.
}

\author{
Maternal and perinatal outcomes associated with COVID-19: A review of \\ the literature.
}

Camila Escobar-Jaramillo ${ }^{1}$, Gabriela Carmach-Ananías ${ }^{1}$, Carlos Kilchemmann Fuentes ${ }^{2}$

${ }^{1}$ Escuela de Obstetricia y Puericultura, Facultad de Ciencias, Universidad Mayor, Chile. (Interna)

${ }^{2}$ Escuela de Obstetricia y Puericultura, Facultad de Ciencias, Universidad Mayor, Chile. Matrón, magíster en Epidemiología Clínica.

*Autor para correspondencia: camila.escobarj@mayor.cl

RECIBIDO: 16 de Noviembre de 2020

APROBADO: 02 de Marzo de 2021

DOI: 10.22370/rev.mat.1.2021.2509

LOS AUTORES DECLARAN NO TENER CONFLICTO DE INTERESES

Palabras claves: Embarazada, Recién Nacido, COVID-19.

Key words: Pregnancy, newborn, COVID-19.

\section{RESUMEN}

Objetivo: Determinar los resultados obstétricos y/o perinatales que se asocian a la infección por COVID-19, a través de una revisión de la literatura.

Metodología: Se realizó una búsqueda avanzada en Pubmed, relacionada con los conceptos paciente e intervención y con los resultados de 67 términos, entre ellos "pregnant", "newborn", "Covid-19", "abortion" y "placental pathology". Se aplicaron como filtro los años 2019 y 2020 y se encontraron 506 estudios que, a su vez, se filtraron por título, resumen y tipo de estudio, seleccionando aquellos cuya muestra fueran embarazadas y/o neonatos diagnosticados con COVID-19, y que reportaran resultados obstétricos y/o perinatales negativos. Finalmente, se seleccionaron 42.

Resultados: Los resultados que destacan por prevalencia o severidad fueron: parto prematuro, patología placentaria, anticuerpos IgM y/o IgG para COVID-19 aumentados en gestantes y recién nacidos, estado fetal no tranquilizador, COVID-19(+) en leche materna, en líquido amniótico o hisopado vaginal, probable transmisión intraparto, ingreso a UCIN, mala perfusión vascular fetal o materna y cesárea por condición materna asociada al virus.

Conclusiones: Sugerimos instaurar medidas de prevención oportunas en las gestantes para evitar el contagio por COVID-19 durante y después de la gestación, con el propósito de evitar el riesgo de complicaciones asociadas al virus, las que conllevan importantes consecuencias para la madre y/o el recién nacido.

\section{ABSTRACT}

Objective: To determine obstetric and perinatal outcomes in COVID-19 infection through a literature review.

Methodology: An advanced search was carried out in Pubmed, with the format: patient, intervention and results with 67 terms, among them: "pregnant", "newborn", "covid-19", "abortion" and "placental pathology". A filter for the year 2019 and 2020 was applied, 506 studies were found that 
were filtered by title, abstract and type of study, selecting those whose sample is pregnant and/or neonates diagnosed with COVID-19, and who report negative obstetric and/or perinatal results. Finally, 42 were selected.

Results: The results that stand out due to their prevalence or severity were: premature delivery, placental pathology, IgM and/or IgG antibodies to COVID-19 increased in pregnant women and newborns, non-reassuring fetal status, COVID-19(+) in breast milk, in liquid amniotic or vaginal swab, probable intrapartum transmission, admission to the NICU, poor fetal or maternal vascular perfusion and caesarean section due to a maternal condition associated with the virus.

Conclusions: We suggest establishing timely prevention measures in pregnant women to avoid contagion by COVID-19 during and after pregnancy, in order to avoid the risk of complications associated with the virus that carry important consequences for the mother and/or newborn.

\section{INTRODUCCIÓN}

La aparición de la nueva infección por coronavirus que se produjo en China, en diciembre de 2019, provocó una pandemia que se ha expandido rápidamente a nivel global, hasta convertirse en una de las amenazas para la salud pública más importantes de los últimos tiempos (1). La aparición de un nuevo coronavirus, denominado SARS-CoV-2, y la enfermedad respiratoria potencialmente mortal que puede producir, COVID-19, se ha extendido con celeridad por todo el mundo. Las epidemias anteriores originadas en infecciones virales emergentes han dado lugar a resultados obstétricos deficientes, incluida la morbilidad y mortalidad materna, la transmisión materno-fetal del virus y las infecciones perinatales y la muerte (3).

Esta nueva enfermedad es la tercera propagación documentada de un coronavirus animal en humanos. Las anteriores epidemias causadas por $\beta$-coronavirus son SARS-CoV y MERS-CoV, que comparten similitudes con SARS-CoV-2 en su estructura genética. Reportaron tasas de letalidad materna de $10 \%$ y $37 \%$ respectivamente, en las que la morbimortalidad materna y perinatal no estuvo exenta (8).
La enfermedad por COVID-19 puede estar asociada a resultados maternos y neonatales adversos en el embarazo, pero hay pocos datos controlados para cuantificar la magnitud de estos riesgos o para caracterizar la epidemiología y los factores de riesgo (12).

Durante el embarazo, las mujeres experimentan cambios inmunológicos y fisiológicos que podrían aumentar su riesgo de enfermedades más graves por infecciones respiratorias (2). Los cambios en los sistemas cardiovascular y respiratorio materno, incluido el aumento de la frecuencia cardíaca, el volumen sistólico, el consumo de oxígeno y la disminución de la capacidad pulmonar, así como el desarrollo de adaptaciones inmunológicas que permiten a la madre tolerar un feto antigénicamente distintivo, aumentan el riesgo para las mujeres embarazadas de desarrollar una enfermedad respiratoria grave (3). Entre las mujeres en edad reproductiva con $\mathrm{CO}-$ VID-19, las embarazadas tienen más probabilidades de ser hospitalizadas y corren mayor riesgo de ingresar a una UCI y recibir ventilación mecánica, en comparación con las no embarazadas, pero su riesgo de muerte es similar (2).

Una pregunta importante que permanece sin respuesta es si el SARS-CoV-2 se puede transmitir de manera vertical y mediante qué mecanismo si ocurre. Esto no solo es un problema importante de salud pública, pues también representa un problema de manejo obstétrico para determinar la atención que reciben las mujeres embarazadas (3).

Por lo anteriormente señalado, el objetivo de esta revisión es determinar resultados obstétricos y perinatales en la infección por COVID-19, a través de una revisión de la literatura.

\section{METODOLOGÍA}

La búsqueda de la literatura se realizó en septiembre de 2020, a través de la base de datos Pubmed y utilizando el formato paciente, intervención y resultado, con un total de 67 términos en inglés de tipo All text y términos Mesh. Asimismo, se utilizó la siguiente estrategia de búsqueda: 1 términos pacientes, 2 términos exposición, 3 resultados, 41 AND 2 AND 3. Se aplicó filtro de año desde 2019 a 2020 y se encontraron 506 estudios, los cuales fueron publicados desde febrero a septiembre del 2020. 
Estos se seleccionaron según título, luego resumen y finalmente lectura de texto completo, quedando 95 artículos.

Los artículos seleccionados fueron distribuidos identificando el tipo de estudio, autor, muestra total y resultados, y se descartaron los estudios secundarios, aquellos que no incluían en su muestra a gestantes o neonatos COVID-19 $(+)$ y que no presentaron resultados obstétricos y/o perinatales. Finalmente se seleccionaron 43 artículos, con los cuales se realizó una tabla con los resultados encontrados en cada uno de ellos, la cual incluyó al autor, la muestra, el número de DOI, los resultados obstétricos y los resultados neonatales.

Figura 1. Diagrama de flujo de identificación de artículos.

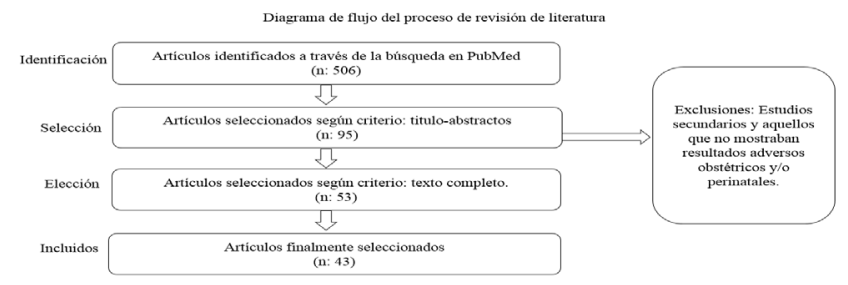

\section{RESULTADOS Y DISCUSIÓN}

Los resultados obstétricos y neonatales se agruparon en una tabla en la que se identifica el número de casos encontrados en los resultados principales de los estudios seleccionados (Tabla 1). Más del 80\% de los artículos seleccionados fueron realizados en China y ningún estudio hizo referencia a características sociodemográficas que pudieran influir en el riesgo de presentar alteraciones asociadas al virus.

El parto prematuro fue el resultado que se asoció con mayor frecuencia a la infección por COVID 19. Resultados similares fueron reportados en revisiones previas (45), en las cuales se reporta una tasa de parto prematuro del 39\%. Aunque el parto prematuro fue principalmente consecuencia de intervenciones electivas, existe una tendencia hacia la prematuridad espontánea (46).

El parto por cesárea también fue un resultado que se observó con mayor frecuencia en las madres infectadas, según la revisión de Zaigham \& Andersson (47), que consideró 18 artículos que informaban datos de 108 embarazos entre el 8 de diciembre de
2019 y el 1 de abril de 2020. Reporta, además, una tasa de cesárea del 91\%. Estos datos concuerdan con los encontrados en la presente revisión de la literatura, donde el parto cesárea tiene una prevalencia elevada, generalmente por causa iatrogénica.

Estos resultados concuerdan también con la revisión de Cabero y cols. (48), que incluyó 33 artículos, con un n de 553 gestantes y 456 partos. Cerca de dos tercios de los nacimientos fueron vía cesárea; no se produjo ninguna muerte materna. Hubo prematuridad en el 22,3\% de los partos y el neonato requirió ingreso en UCI en el 38,3\% de los casos. Solo se informó de una muerte neonatal $(0,4 \%)$ y 13 casos de COVID-19 neonatal (3,4\%). La información disponible no permite asegurar que la transmisión se produjera por vía transplacentaria.

Los resultados reportados en otras publicaciones son comparables con los presentados en la presente revisión. La mayoría de los efectos adversos reportados se asemejan, posicionándose como principal el parto prematuro. La vía del parto por cesárea -de igual manera- es un factor importante, al dar cuenta de que en la mayoría de los casos se opta por esta vía de parto a modo de asegurar el bienestar fetal y materno.

En esta revisión de la literatura no fue posible establecer medidas de riesgo, debido principalmente a la heterogeneidad de las variables de resultado.

\section{CONCLUSIONES}

En la actualidad, no existe evidencia que demuestre la transmisión vertical del virus SARS-COV2 durante la gestación, periodo intraparto ni a través de la lactancia materna, pero hay antecedentes que demuestran resultados adversos de la enfermedad sobre la gestación y el recién nacido, siendo los más frecuentes y relevantes el parto prematuro y el deterioro placentario, patologías que repercuten de forma grave sobre los recién nacidos.

Es notable el predominio de alteraciones de tipo inflamatorio y de la perfusión sanguínea uteroplacentaria en gestantes infectadas, las cuales generan la base para una cascada de complicaciones madrehijo como son el parto prematuro, la neumonía, el crecimiento restrictivo fetal, la rotura prematura de 
membranas, la corioamnionitis, el síndrome hipertensivo del embarazo o el bajo peso de nacimiento, entre otras.

Teniendo en cuenta que el COVID-19 tiene un potencial patógeno para causar resultados adversos maternos o perinatales graves, sugerimos una búsqueda activa del virus SARS-COV-2 en la población de embarazadas, mediante una evaluación universal a todas las gestantes -previa al parto- y a sus recién nacidos, con PCR de hisopado nasofaríngeo.

El tener conocimiento de los efectos negativos producidos por la infección del virus en la gestación permite orientar el quehacer de los profesionales de la salud en el contexto actual de pandemia y crear medidas preventivas y de autocuidado para la embarazada, diada y su entorno familiar, como, también, definir y aplicar protocolos y normativas locales en cada establecimiento de salud, para la protección y manejo adecuados de casos confirmados y sospechosos de gestantes COVID-19 (+). Además, para reducir la aparición de enfermedades graves, se debe asesorar a las mujeres embarazadas sobre el riesgo potencial de la enfermedad por COVID-19 y se debe enfatizar en las medidas de prevención.

\section{REFERENCIAS}

1. Chen, L., Li, Q., Zheng, D., Jiang, H., Wei, Y., Zou, L., ... Qiao, J. (17 de Abril de 2020). Clinical Characteristics of Pregnant Women with COVID-19 in Wuhan, China. NEJM Group Public Health Emergency Collection, 382(25). doi:10.1056/NEJMc2009226

2. llington, S., Strid, P., Tong, V., Woodworth, K., Galang, R., Zambrano, L., . . G Gilboa, S. (Junio de 2020). Characteristics of Women of Reproductive Age with Laboratory-Confirmed SARS-CoV-2 Infection by Pregnancy Status - United States, January 22-June 7, 2020. MMWR. Morbidity and mortality weekly report, 69(25), 769-775. doi:10.15585/mmwr. mm6925a1

3. Schwartz, D. (17 de Marzo de 2020). An Analysis of 38 Pregnant Women with COVID-19, Their Newborn Infants, and Maternal-Fetal Transmission of SARS-CoV-2: Maternal Coronavirus Infections and Pregnancy Outcomes. Arch Pathol Lab Med, 144(7), 799-805. doi:10.5858/arpa.2020-0901-SA
4. Cao, D., Heng, Y., Sun, G., Chen, J., Wei, X., Tang, F., . . . Zhao, Y. (1 de Junio de 2020). Clinical analysis of ten pregnant women with COVID-19 in Wuhan, China: A retrospective study. International Journal of Infectious Diseases, 95, 294-300. doi:10.1016/j.ijid.2020.04.047

5. Vlachodimitropoulou, E., Vivanti, A., Shehata, N., Benachi, A., \& Kinga, A. (13 de Abril de 2020). COVID-19 and acute coagulopathy in pregnancy. Journal of Thrombosis and Haemostasis, 18(7), 16481652. doi:10.1111/jth.14856

6. Ferrazi, E., Frigerio, L., Savasi, V., Vergani, P., Prefumo, F., Barresi, S., . . . Villa, U. (28 de Mayo de 2020). Vaginal delivery in SARS-CoV-2-infected pregnant women in Northern Italy: a retrospective analysis. BJOG : an international journal of obstetrics and gynaecology, 1-6. doi:10.1111/1471-0528.16278

7. Zhu, H., Wang, L., Fang, C., Peng, S., Zhang, L., Chang, G., .. . Zhou, W. (Febrero de 2020). Clinical analysis of 10 neonates born to mothers with 2019nCoV pneumonia. Translational Pediatrics, 9(1), 5160. doi:10.21037/tp.2020.02.06

8. Wu, Y., Liu, C., Dong, L., Zhang, C., Chen, Y., Liu, J., ... Huang, H. (5 de Mayo de 2020). Coronavirus disease 2019 among pregnant Chinese women: case series data on the safety of vaginal birth and breastfeeding. BJOG: An International Journal of Obstetrics \& Gynaecology, 127(9), 1109-1115. doi:10.1111/14710528.16276

9. Zamaniyan, M., Ebadi, A., Aghajanpoor, S., Rahmani, Z., Haghshenas, M., \& Azizi, S. (17 de Abril de 2020). Preterm delivery in pregnant woman with critical COVID-19 pneumonia and vertical transmission. Wiley Public Health Emergency Collection. doi:10.1002/pd.5713

10. Kirstman, M., Diambomba, Y., Poutanen, S., Malinowski, A., Vlachodimitropoulou, E., Parks, T., . .. Shah, P. (15 de Junio de 2020). Probable congenital SARS-CoV-2 infection in a neonate born to a woman with active SARS-CoV-2 infection. CMAJ, 192(24), E647- E650. doi:10.1503/cmaj.200821 
11. Zeng, H., Xu, C., Fan, J., Tang, Y., Deng, Q., Zhang, W., \& Long, X. (26 de Marzo de 2020). Antibodies in Infants Born to Mothers With COVID-19 Pneumonia. JAMA Network, 323(18), 1848-1849. doi:10.1001/jama.2020.4861

12. Brandt, J., Hill, J., Reddy, A., Schuster, M., Patrick, H., Rosen, T., . . . Ananth, C. (25 de Septiembre de 2020). Epidemiology of COVID-19 in Pregnancy: Risk Factors and Associations with Adverse Maternal and Neonatal Outcomes. American Journal of Obstetrics and Gynecology. doi:10.1016/j.ajog.2020.09.043

13. Baergen, R., \& Heller, D. (12 de Mayo de 2020). Placental Pathology in COVID-19 Positive Mothers: Preliminary Findings. Pediatric and evelopmental Pathology, 23(3), 177-180. doi:10.1177/1093526620925569

14. Kuhrt, K., McMicking, J., Nanda, S., NelsonPiercy, C., Shennan, A. (8 de mayo de 2020). Placental abruption in a twin pregnancy at 32 weeks' gestation complicated by coronavirus disease 2019 without vertical transmission to the babies. American journal of obstetrics \& gynecology MFM, 2(3), 100135. doi: 10.1016/j.ajogmf.2020.100135

15. Pierce-Williams, R., Burd J, Felder L, et al. (8 de mayo de 2020). Clinical course of severe and critical coronavirus disease 2019 in hospitalized pregnancies: a United States cohort study. Am J Obstet Gynecol MFM. 2020;2(3):100134. doi: 10.1016/j.ajogmf.2020.100134

16. Penfield, C. A., Brubaker, S. G., Limaye, M. A., Lighter, J., Ratner, A. J., Thomas, K. M., Meyer, J. A., \& Roman, A. S. (8 de mayo de 2020). Detection of severe acute respiratory syndrome coronavirus 2 in placental and fetal membrane samples. American journal of obstetrics \& gynecology MFM, 2(3), 100133. doi: 10.1016/j.ajogmf.2020.100133

17. Foong, K., Bandi, S., Bird, P. W., \& Wei-Tze Tang, J. (Julio de 2020). COVID-19 in Neonates and Infants: Progression and Recovery. The Pediatric infectious disease journal, 39(7), e140-e142. 10.1097/ INF.0000000000002738.
18. Vallejo, V., \& Ilagan, J. G. (Julio de 2020). A Postpartum Death Due to Coronavirus Disease 2019 (COVID-19) in the United States. Obstetrics and gynecology, 136(1), 52-55. doi: 10.1097/ AOG.0000000000003950

19. Blauvelt, C. A., Chiu, C., Donovan, A. L., Prahl, M., Shimotake, T. K., George, R. B., Schwartz, B. S., Farooqi, N. A., Ali, S. S., Cassidy, A., Gonzalez, J. M., \& Gaw, S. L. (Julio de 2020). Acute Respiratory Distress Syndrome in a Preterm Pregnant Patient With Coronavirus Disease 2019 (COVID-19). Obstetrics and gynecology, 136(1), 46-51.doi: 10.1097/ AOG.0000000000003949

20. Martinelli, I., Ferrazzi, E., Ciavarella, A., Erra, R., Iurlaro, E., Ossola, M., Lombardi, A., Blasi, F., Mosca, F., \& Peyvandi, F. (Abril de 2020). Pulmonary embolism in a young pregnant woman with COVID-19. Thrombosis research, 191, 36-37.doi: 10.1016/j.thromres.2020.04.022

21. Kuusela, A., Nazir, M., Gimovsky, M. (3 de Abril de 2020). Two cases of coronavirus 2019-related cardiomyopathy in pregnancy. American journal of obstetrics \& gynecology MFM, 2(2), 100113. doi: 10.1016/j.ajogmf.2020.100113

22. Xu, L., Yang, Q., Shi, H., Lei, S., Liu, X., Zhu, Y., Wu, Q., Ding, X., Tian, Y., Hu, Q., Chen, F., Geng, Z., Zeng, X., Lin, L., Cai, X., Wu, M., Wang, Z., Wang, Z., Xia, G., \& Wang, L. (28 de abril de 2020). Clinical presentations and outcomes of SARS-CoV-2 infected pneumonia in pregnant women and health status of their neonates. Science bulletin, 65(18), 1537-1542. doi: 10.1016/j.scib.2020.04.040

23. Patanè, L., Morotti, D., Giunta, M. R., Sigismondi, C., Piccoli, M. G., Frigerio, L., Mangili, G., Arosio, M., \& Cornolti, G. (18 de mayo de 2020). Vertical transmission of coronavirus disease 2019: severe acute respiratory syndrome coronavirus 2 RNA on the fetal side of the placenta in pregnancies with coronavirus disease 2019-positive mothers and neonates at birth. American journal of obstetrics \& gynecology MFM, 2(3), 100145. doi: 10.1016/j.ajogmf.2020.100145. 
24. Hu, X., Gao, J., Luo, X., Feng, L., Liu, W., Chen, J., Benachi, A., De Luca, D., \& Chen, L. (Julio de 2020). Severe Acute Respiratory Syndrome Coronavirus 2 (SARS-CoV-2) Vertical Transmission in Neonates Born to Mothers With Coronavirus Disease 2019 (COVID-19) Pneumonia. Obstetrics and gynecology, 136(1), 65-67. doi: 10.1097/AOG.0000000000003926

25. Sentilhes, L., De Marcillac, F., Jouffrieau, C., Kuhn, P., Thuet, V., Hansmann, Y., Ruch, Y., FafiKremer, S., \& Deruelle, P. (15 de junio de 2020). Coronavirus disease 2019 in pregnancy was associated with maternal morbidity and preterm birth. American journal of obstetrics and gynecology, S00029378(20)30639-6. Advance online publication.doi: 10.1016/j.ajog.2020.06.022.

26. Alzamora, M. C., Paredes, T., Caceres, D., Webb, C. M., Valdez, L. M., \& La Rosa, M. (18 de abril de 2020). Severe COVID-19 during Pregnancy and Possible Vertical Transmission. American journal of perinatology, 37(8), 861-865. doi: 10.1055/s-00401710050

27. Khan, S., Jun, L., Nawsherwan, Siddique, R., Li, Y., Han, G., Xue, M., Nabi, G., \& Liu, J. (8 de abril de 2020). Association of COVID-19 with pregnancy outcomes in health-care workers and general women. Clinical microbiology and infection: the official publication of the European Society of Clinical Microbiology and Infectious Diseases, 26(6), 788-790. doi: 10.1016/j.cmi.2020.03.034

28. Hang, Z. J., Yu, X. J., Fu, T., Liu, Y., Jiang, Y., Yang, B. X., \& Bi, Y. (18 de junio de 2020). Novel coronavirus infection in newborn babies aged $<28$ days in China. The European respiratory journal, 55(6), 2000697. doi: 10.1183/13993003.00697-2020

29. Chen, Y., Peng, H., Wang, L., Zhao, Y., Zeng, L., Gao, H., \& Liu, Y. (16 de marzo de 2020). Infants Born to Mothers With a New Coronavirus (COVID-19). Frontiers in pediatrics, 8, 104. doi: 10.3389/ fped.2020.00104

30. Zeng, L. K., Tao, X. W., Yuan, W. H., Wang, J., Liu, X., \& Liu, Z. S. (2 de abril de 2020). Zhonghua er ke za zhi $=$ Chinese journal of pediatrics, 58(4), 279280. doi: 10.3760/cma.j.cn112140-20200212-00081
31. Kamali M., Jafari, N., \& Eftekhari, K. (1 de abril de 2020). Novel coronavirus in a 15-day-old neonate with clinical signs of sepsis, a case report. Infectious diseases (London, England), 52(6), 427429. doi: $10.1080 / 23744235.2020 .1747634$

32. Wang, Z., Wang, Z., \& Xiong, G. (8 de junio de 2020). Clinical characteristics and laboratory results of pregnant women with COVID-19 in Wuhan, China. International journal of gynaecology and obstetrics: the official organ of the International Federation of Gynaecology and Obstetrics, 150(3), 312-317. Advance online publication. doi: 10.1002/ijgo.13265

33. Dong, L., Tian, J., He, S., Zhu, C., Wang, J., Liu, C., \& Yang, J. (12 de mayo de 2020). Possible Vertical Transmission of SARS-CoV-2 From an Infected Mother to Her Newborn. JAMA, 323(18), 1846-1848. doi: 10.1001/jama.2020.4621

34. Liu, D., Li, L., Wu, X., Zheng, D., Wang, J., Yang, L., \& Zheng, C. (Julio de 2020). Pregnancy and Perinatal Outcomes of Women With Coronavirus Disease (COVID-19) Pneumonia: A Preliminary Analysis. AJR. American journal of roentgenology, 215(1), 127-132. doi: 10.2214/AJR.20.23072

35. Chen, R., Zhang, Y., Huang, L., Cheng, B. H., Xia, Z. Y., \& Meng, Q. T. (16 de marzo 2020). Safety and efficacy of different anesthetic regimens for parturients with COVID-19 undergoing Cesarean delivery: a case series of 17 patients. Sécurité et efficacité de différents modes d'anesthésie pour des parturientes infectées par la COVID-19 accouchant par césarienne : une série de 17 cas. Canadian journal of anaesthesia $=$ Journal canadien d'anesthesie, 67(6), 655-663. doi: 10.1007/s12630-020-01630-7

36. Chen, S., Huang, B., Luo, D. J., Li, X., Yang, F., Zhao, Y., Nie, X., \& Huang, B. X. (8 de mayo de 2020). Zhonghua bing li xue za zhi $=$ Chinese journal of pathology, 49(5), 418-423. doi: 10.3760/ cma.j.cn112151-20200225-00138

37. Shanes, E. D., Mithal, L. B., Otero, S., Azad, H. A., Miller, E. S., \& Goldstein, J. A. (12 de mayo de 2020). Placental pathology in COVID-19. medRxiv : the preprintserverforhealth sciences, 2020.05.08.20093229. doi: 10.1101/2020.05.08.20093229 
38. Prabhu, M., Cagino, K., Matthews, K. C., Friedlander, R. L., Glynn, S. M., Kubiak, J. M., Yang, Y. J., Zhao, Z., Baergen, R. N., DiPace, J. I., Razavi, A. S., Skupski, D. W., Snyder, J. R., Singh, H. K., Kalish, R. B., Oxford, C. M., \& Riley, L. E. (13 de agosto de 2020). Pregnancy and postpartum outcomes in a universally tested population for SARS-CoV-2 in New York City: a prospective cohort study. BJOG : an international journal of obstetrics and gynaecology, 127(12), 1548-1556. doi:10.1111/1471-0528.16403

39. Ferraiolo, A., Barra, F., Kratochwila, C., Paudice, M., Vellone, V. G., Godano, E., Varesano, S., Noberasco, G., Ferrero, S., \& Arioni, C. (22 de junio de 2020). Report of Positive Placental Swabs for SARSCoV-2 in an Asymptomatic Pregnant Woman with COVID-19. Medicina (Kaunas, Lithuania), 56(6), 306. doi:10.3390/medicina56060306.

40. Nawsherwan, Khan, S., Nabi, G., Fan, C., \& Wang, S. (22 de junio de 2020). Impact of COVID-19 Pneumonia on Neonatal Birth Outcomes. Indian journal of pediatrics, 87(8), 645-646. doi: 10.1007/ s12098-020-03372-2

41. Mendoza, M., Garcia-Ruiz, I., Maiz, N., Rodo, C., Garcia-Manau, P., Serrano, B., Lopez-Martinez, R. M., Balcells, J., Fernandez-Hidalgo, N., Carreras, E., \& Suy, A. (21 de junio de 2020). Pre-eclampsia-like syndrome induced by severe COVID-19: a prospective observational study. BJOG: an international journal of obstetrics and gynaecology, 127(11), 1374-1380. doi: 10.1111/1471-0528.16339

42. Futterman, I., Toaff, M., Navi, L., \& Clare, C. A. (18 de junio de 2020). COVID-19 and HELLP: Overlapping Clinical Pictures in Two Gravid Patients. AJP reports, 10(2), e179-e182. doi: 10.1055/s-00401712978

43. Grob, R., Carina, C., Muller, J., Stenger, S., Steinhart, K., Kirchhoff, F., \& Munch. (21 de Mayo de 2020). Detection of SARS-CoV-2 in human breastmilk. The Lancet, 395, 1751-1758. doi:10.1016/S01406736(20)31181-8

44. Hasbun, J., \& Hasbun, A. (2000). Infeccion y parto prematuro: enlace epidemiológico y bioquímico. Revista Chilena De Infectologia, 17(1), 7-17. doi:10.4067/S0716-10182000000100002
45. Dashraath, P., Lin, J., Xian, M., Choolani, M., Mattar, C., \& Lin, S. Coronavirus disease 2019 (COVID-19) pandemic and pregnancy. Special Report, 222, 521-531. doi:10.1016/j.ajog.2020.03.021

46. Della, A., Rizzo, R., Pilu, G., \& Simonazzi, G. (17 de Abril de 2020). Coronavirus disease 2019 during pregnancy: a systematic review of reported cases. Systematic Reviews, 223. doi:10.1016/j. ajog.2020.04.013

47. Zaigham, M., \& Andersson, O. Maternal and perinatal outcomes with COVID-19: A systematic review of 108 pregnancies. Acta Obstetricia et Gynecologica Scandinavica, 99. doi:10.1111/aogs.13867

48. Cabero, M., Gómez, I., Dierssen, T., \& Llorca, J. (Agosto de 2020). Infección por SARS-CoV-2 en el embarazo y posibilidad de transmisión al neonato: una revisión sistemática. Elsevier Public Health Emergency Collection, 40-47. doi:10.1016/j.semerg.2020.06.011 
Tabla 1: Resultados obstétricos y neonatales asociados a la infección por COVID-19

\begin{tabular}{|c|c|c|c|c|}
\hline $\begin{array}{l}\text { Autores, año } \\
\text { y ciudad }\end{array}$ & Título del estudio & Muestra & $\begin{array}{c}\text { Resultados obstétricos y } \\
\text { número de casos }\end{array}$ & $\begin{array}{c}\text { Resultados neonatales y } \\
\text { número de casos }\end{array}$ \\
\hline $\begin{array}{l}\text { Cao, D., et al. } \\
2020 \\
\text { Wuhan, China. }{ }^{(4)}\end{array}$ & $\begin{array}{l}\text { Clinical analysis of ten } \\
\text { pregnant women with } \\
\text { COVID-19 in Wuhan, } \\
\text { China: A retrospective } \\
\text { study. }\end{array}$ & $\begin{array}{l}10 \text { gestantes }\left(1 \text { ge}^{-}\right. \\
\text {melar) entre } 33+6 \\
\text { a } 40+5 \text { semanas. }\end{array}$ & $\begin{array}{l}\text { Cesárea intraparto por sufrimien- } \\
\text { to fetal agudo: } 2 \text {. } \\
\text { Cesáreas electivas por cesárea an- } \\
\text { terior o DPPNI o PE o gemelar: } 6 . \\
\text { Rotura prematura de membranas: } \\
4 . \\
\text { Preeclampsia: } 3 \text {. } \\
\text { DPPNI: } 1 \text {. }\end{array}$ & RN Pretérmino: 4. \\
\hline $\begin{array}{l}\text { Vlachodimitro- } \\
\text { poulou, E., Vi- } \\
\text { vanti, A., Sheha- } \\
\text { ta, N., Benachi, } \\
\text { A., \& Kinga, A. } \\
\text { 2020. Toronto, } \\
\text { Canadá.(5) }\end{array}$ & $\begin{array}{l}\text { COVID-19 and acute } \\
\text { coagulopathy in preg- } \\
\text { nancy. }\end{array}$ & $\begin{array}{l}2 \text { gestantes. } \\
\text { Caso 1: G2P1 35+5 } \\
\text { semanas, } 40 \text { años. } \\
\text { Caso 2: Primigesta } \\
\text { 35+2 semanas, } 23 \\
\text { años. }\end{array}$ & $\begin{array}{l}\text { Caso 1: Cesárea por probable im- } \\
\text { posibilidad de anestesia neuroa- } \\
\text { xial por coagulopatía y hemorra- } \\
\text { gia postparto } \\
\text { Caso 2: Cesárea de emergencia } \\
\text { por estado fetal no tranquiliza- } \\
\text { dor más coagulopatía progresiva y } \\
\text { transaminitis. }\end{array}$ & $\begin{array}{l}\text { RN pretérmino: } 2 . \\
\text { Caso } 2 \text { : APGAR de } 4,2,7 \text { a } 1,5 \\
\text { y } 10 \text { minutos, respectivamente. }\end{array}$ \\
\hline $\begin{array}{l}\text { Ferrazi, E., et } \\
\text { al. 2020. Milán, } \\
\text { Italia. }{ }^{(6)}\end{array}$ & $\begin{array}{l}\text { Vaginal delivery in } \\
\text { SARS-CoV-2-infected } \\
\text { pregnant women in } \\
\text { Northern Italy: a ret- } \\
\text { rospective analysis. }\end{array}$ & $\begin{array}{l}\text { Muestra: } 42 \text { puér- } \\
\text { peras dentro de } \\
\text { las } 36 \text { horas pos- } \\
\text { tparto. }\end{array}$ & $\begin{array}{l}\text { Cesáreas: } 18 \text {, de ellas } 11 \text { cesáreas } \\
\text { electivas por empeoramiento de } \\
\text { síntomas. } \\
\text { Inducción y parto vaginal: } 3 \text {. } \\
\text { Parto prematuro: } 11 \text {, de ellos, } 5 \text { fue- } \\
\text { ron espontáneos. } \\
\text { Hemorragia postparto: } 1 \text {. }\end{array}$ & $\begin{array}{l}\text { RN COVID-19(+):1 de parto va- } \\
\text { ginal con aislamiento inmediato } \\
\text { postparto. Tuvo síntomas gas- } \\
\text { trointestinales y respiratorios. } \\
\text { Requirió de ingreso a UCIN y } \\
\text { un día de ventilación mecánica. } \\
2 \text { RN fueron (+) luego del diag- } \\
\text { nóstico de COVID-19 en } 2 \text { mu- } \\
\text { jeres postparto que no usaron } \\
\text { mascarilla. } \\
\text { Ingreso a UCIN: } 3 \text {. } \\
\text { RN pretérmino: } 2 \text { con APGAR }<7 \\
\text { a los } 5 \text { minutos. }\end{array}$ \\
\hline
\end{tabular}




\begin{tabular}{|c|c|c|c|c|}
\hline $\begin{array}{c}\text { Autores, año } \\
\text { y ciudad }\end{array}$ & Título del estudio & Muestra & $\begin{array}{l}\text { Resultados obstétricos y } \\
\text { número de casos }\end{array}$ & $\begin{array}{l}\text { Resultados neonatales y } \\
\text { número de casos }\end{array}$ \\
\hline $\begin{array}{l}\text { Zhu, H., et al. } \\
2020 . \text { Wuhan, } \\
\text { China. }{ }^{(7)}\end{array}$ & $\begin{array}{l}\text { Clinical analysis of } \\
10 \text { neonates born to } \\
\text { mothers with 2019- } \\
\text { nCoV pneumonia. }\end{array}$ & $\begin{array}{l}9 \text { gestantes, } 1 \text { ge- } \\
\text { melar. }\end{array}$ & $\begin{array}{l}\text { Estado fetal no tranquilizador: } 6 . \\
\text { Rotura prematura de membranas: } \\
3 . \\
\text { Polihidramnios: } 1 . \\
\text { Oligohidramnios: } 1 . \\
\text { Torsión de cordón: } 1 . \\
\text { Cordón coloide: } 1 . \\
\text { Placenta previa: } 1 \text {. }\end{array}$ & $\begin{array}{l}\text { Pequeño para la edad gesta- } \\
\text { cional: } 2 \text {. Grande para la edad } \\
\text { gestacional: } 1 \text {. } \\
\text { RN con síntomas gastrointesti- } \\
\text { nales: } 4 \text {. Rx de tórax anormal: } 4 \text {. } \\
\text { Síndrome de distrés respirato- } \\
\text { rio: } 2 \text {. } \\
\text { Trombocitopenia complicada: } \\
2 . \\
\text { Alteración de la función hepá- } \\
\text { tica: } 2,1 \text { de ellos fue prematuro } \\
\text { y paso a falla multiorgánica con } \\
\text { CID y fallece al } 9^{\circ} \text { día. } \\
\text { El otro prematuro requirió oxi- } \\
\text { genoterapia y transfusiones, se } \\
\text { curó a los } 15 \text { días. }\end{array}$ \\
\hline $\begin{array}{l}\text { Wu, I., et al. } \\
2020 . \text { Wuhan, } \\
\text { China. }{ }^{(8)}\end{array}$ & $\begin{array}{l}\text { Coronavirus disease } \\
2019 \text { among pregnant } \\
\text { Chinese women: case } \\
\text { series data on the } \\
\text { safety of vaginal birth } \\
\text { and breastfeeding. }\end{array}$ & $\begin{array}{l}13 \text { gestantes de } \\
\text { entre } 5 \text { a } 38 \text { sema- } \\
\text { nas. }\end{array}$ & $\begin{array}{l}\text { Demostración de COVID-19 en he- } \\
\text { ces: } 1 \text { de } 9 \text { muestras. } \\
\text { Estado fetal no tranquilizador con } \\
\text { parto prematuro: } 1 \text {. } \\
\text { Parto prematuro espontáneo: } 1 . \\
\text { Cesáreas a causa del descono- } \\
\text { cimiento y miedo de la probable } \\
\text { transmisión vertical del virus: } 3 \text {. } \\
\text { Estudio de leche materna para } \\
\text { COVID-19 (+): } 1 \text { de } 3 \text {, positivo al } \\
\text { primer día postparto, negativo al } \\
\text { tercer día. }\end{array}$ & $\begin{array}{l}\text { RN pretérmino: } 2 \text {. } \\
\text { Neumonía neonatal: } 2 \text {. } \\
\text { Grande para la edad gestacio- } \\
\text { nal: } 1 . \\
\text { Seroconversión de IgM e IgG: } 1 .\end{array}$ \\
\hline $\begin{array}{l}\text { Zamaniyan, M., } \\
\text { Ebadi, A., Agha- } \\
\text { janpoor, S., Rah- } \\
\text { mani, Z., Haghs- } \\
\text { henas, M., \& } \\
\text { Azizi, S. } 2020 . \\
\text { Sari, Irán. }\end{array}$ & $\begin{array}{l}\text { Preterm delivery, ma- } \\
\text { ternal death, and ver- } \\
\text { tical transmission in a } \\
\text { pregnant woman with } \\
\text { COVID-19 infection. }\end{array}$ & $\begin{array}{l}1 \text { gestante de } 32 \\
\text { semanas. }\end{array}$ & $\begin{array}{l}\text { Cesárea por condición materna } \\
\text { asociada al virus. } \\
\text { Parto prematuro. } \\
\text { Muestra de líquido amniótico CO- } \\
\text { VID-19 (+). } \\
\text { Muerte materna. }\end{array}$ & $\begin{array}{l}\text { Ingreso a UCIN. } \\
\text { Bajo peso de nacimiento. } \\
\text { RN COVID-19 }(+) \text { a las } 24 \text { horas } \\
\text { de vida. }\end{array}$ \\
\hline
\end{tabular}




\begin{tabular}{|c|c|c|c|c|}
\hline $\begin{array}{l}\text { Autores, año } \\
\text { y ciudad }\end{array}$ & Título del estudio & Muestra & $\begin{array}{c}\text { Resultados obstétricos y } \\
\text { número de casos }\end{array}$ & $\begin{array}{l}\text { Resultados neonatales y } \\
\text { número de casos }\end{array}$ \\
\hline $\begin{array}{l}\text { Kirstman, M., et } \\
\text { al. 2020. Onta- } \\
\text { rio, Canadá. }{ }^{(10)}\end{array}$ & $\begin{array}{l}\text { Probable congenital } \\
\text { SARS-CoV-2 infection } \\
\text { in a neonate born to } \\
\text { a woman with active } \\
\text { SARS-CoV-2 infection. }\end{array}$ & $\begin{array}{l}\text { Muestra: } 1 \text { gestan- } \\
\text { te G2P1 con } 35+5 \\
\text { semanas, } \\
40 \text { años. }\end{array}$ & $\begin{array}{l}\text { Presencia del virus COVID -19 en } \\
\text { leche materna: }(+) \\
\text { Presencia del virus COVID-19 en } \\
\text { hisopado vaginal: }(+) \\
\text { Cesárea semi urgente por estado } \\
\text { materno empeorado asociado a la } \\
\text { coagulopatía a causa del virus. } \\
\text { La placenta mostró múltiples áreas } \\
\text { de infiltración por células inflama- } \\
\text { torias e infarto temprano extenso. }\end{array}$ & $\begin{array}{l}\text { PCR COVID-19 }(+) \text { en plasma. } \\
\text { PCR COVID-19 }(+) \text { en heces. } \\
\text { Hipoglicemia. } \\
\text { Hipotermia. } \\
\text { Ingreso a UCIN. } \\
\text { Neutropenia } \\
\text { Alteración de la función hepá- } \\
\text { tica. } \\
\text { (RN con aislamiento inmediato } \\
\text { postparto) }\end{array}$ \\
\hline $\begin{array}{l}\text { Zeng, H., et al. } \\
2020 \text {. Wuhan, } \\
\text { China. }{ }^{(11)}\end{array}$ & $\begin{array}{l}\text { Antibodies in Infants } \\
\text { Born to Mothers With } \\
\text { COVID-19 Pneumonia. }\end{array}$ & $\begin{array}{l}\text { Muestra: } 6 \text { ges- } \\
\text { tantes }\end{array}$ & $\begin{array}{l}\text { Cesáreas: todas. } \\
\text { IgG y/o IgM elevadas para CO- } \\
\text { VID-19 sin otros patógenos. }\end{array}$ & $\begin{array}{l}\text { Todos los RN con anticuerpos } \\
\text { aumentados en suero ( } 5 \text { de IgG } \\
\text { y } 2 \text { de IgM). } \\
\text { Citocina inflamatoria interleu- } \\
\text { kina- } 6 \text { aumentada: todos. }\end{array}$ \\
\hline $\begin{array}{l}\text { Brandt, j., et } \\
\text { al. 2020. New } \\
\text { B r un s w i c k, } \\
\text { USA. }{ }^{(12)}\end{array}$ & $\begin{array}{l}\text { Epidemiology of coro- } \\
\text { navirus disease } 2019 \\
\text { in pregnancy: risk fac- } \\
\text { tors and associations } \\
\text { with adverse mater- } \\
\text { nal and neonatal out- } \\
\text { comes. }\end{array}$ & $\begin{array}{l}\text { Muestra: } 61 \text { ges- } \\
\text { tantes entre } 16 \text { y } \\
41 \text { semanas entre } \\
11 \text { marzo y } 11 \text { junio. } \\
\text { Emparejaron } 2: 1 .\end{array}$ & $\begin{array}{l}\text { Parto prematuro: } 13 \text {. } \\
\text { Corioamnionitis: } 2 \text {. } \\
\text { MEFI en categoría } 2 \text { persistente: } 6 \\
\text { Preeclampsia: } 6\end{array}$ & $\begin{array}{l}\text { Ingreso a UCIN: } 53 . \\
\text { Síndrome de distrés respirato- } \\
\text { rio: } 5 . \\
\text { Hemorragia interventricular: } 1 . \\
\text { Bajo peso de nacimiento: varios } \\
\text { (no identifica con claridad). } \\
\text { Muerte neonatal: } 1 .\end{array}$ \\
\hline $\begin{array}{l}\text { Baergen, R., \& } \\
\text { Heller, D. 2020. } \\
\text { New York, USA. } \\
\text { (13) }\end{array}$ & $\begin{array}{l}\text { Placental Pathology } \\
\text { in COVID-19 Positive } \\
\text { Mothers: Preliminary } \\
\text { Findings. }\end{array}$ & $\begin{array}{l}20 \text { gestantes de } \\
\text { entre } 32+2 \text { y } 40+4 \\
\text { semanas. }\end{array}$ & $\begin{array}{l}\text { Lesiones de mala perfusión vascu- } \\
\text { lar materna: } 5 \text {. } \\
\text { Mala perfusión vascular fetal de } \\
\text { bajo grado (depósito de fibrina en } \\
\text { íntima del vaso o trombosis de ve- } \\
\text { llosidades coriónicas): } 9 . \\
\text { Corioamnionitis y funisitis aguda: } \\
1 . \\
\text { Infarto placentario masivo: } 1 \text {. } \\
\text { Corangioma:1. }\end{array}$ & $\begin{array}{l}\text { No se describen o no se encon- } \\
\text { traron alteraciones. }\end{array}$ \\
\hline
\end{tabular}




\begin{tabular}{|c|c|c|c|c|}
\hline $\begin{array}{l}\text { Autores, año } \\
\text { y ciudad }\end{array}$ & Título del estudio & Muestra & $\begin{array}{l}\text { Resultados obstétricos y } \\
\text { número de casos }\end{array}$ & $\begin{array}{l}\text { Resultados neonatales y } \\
\text { número de casos }\end{array}$ \\
\hline $\begin{array}{l}\text { Kuhrt, K., Mc- } \\
\text { Micking, J., } \\
\text { Nanda, S., Nel- } \\
\text { son-Piercy, C., } \\
\text { Shennan, A. } \\
\text { 2020. Londres, } \\
\text { Inglaterra. }{ }^{(14)}\end{array}$ & $\begin{array}{l}\text { Placental abruption } \\
\text { in a twin pregnancy } \\
\text { at } 32 \text { weeks' gesta- } \\
\text { tion complicated by } \\
\text { coronavirus disease } \\
2019 \text { without vertical } \\
\text { transmission to the } \\
\text { babies. }\end{array}$ & $\begin{array}{l}1 \text { gestación geme- } \\
\text { lar biamniótica } \\
\text { monocorial de } 32 \\
\text { semanas. }\end{array}$ & $\begin{array}{l}\text { Desprendimiento prematuro de } \\
\text { placenta normo inserta. } \\
\text { Parto prematuro. } \\
\text { Cesárea de emergencia por metro- } \\
\text { rragia ante parto. } \\
\text { Hipoperfusión placentaria. } \\
\text { Maduración vellositaria acelerada } \\
\text { (hipoperfusión leve). }\end{array}$ & $\begin{array}{l}\text { Necesidad de reanimación neo- } \\
\text { natal: ambos. } \\
\text { Ingreso a UCIN: ambos. }\end{array}$ \\
\hline $\begin{array}{l}\text { P i e r c e - W i } 1 \text { - } \\
\text { liams, R. } \\
\text { 2020. New York, } \\
\text { USA. }^{(15)}\end{array}$ & $\begin{array}{l}\text { Clinical course of se- } \\
\text { vere and critical coro- } \\
\text { navirus disease } 2019 \text { in } \\
\text { hospitalized pregnan- } \\
\text { cies: a United States } \\
\text { cohort study. }\end{array}$ & $\begin{array}{l}64 \text { gestantes de } \\
\text { promedio } 30+-6 \\
\text { semanas. } \\
44 \text { con enferme- } \\
\text { dad grave y } 20 \text { con } \\
\text { enfermedad críti- } \\
\text { ca por Covid-19. }\end{array}$ & $\begin{array}{l}\text { Enfermedad grave: } \\
\text { Promedio de edad gestacional al } \\
\text { parto: } 37 \text { semanas. } \\
\text { 15/44 parto durante curso de CO- } \\
\text { VID-19. } \\
\text { Enfermedad crítica: } \\
\text { 17/20 parto durante curso de CO- } \\
\text { VID-19. } \\
\text { Parto/cesárea por estado mater- } \\
\text { no: } 22 . \\
\text { Parto/cesárea por estado fetal: } 3 . \\
\text { Parto/cesárea por causa obstétri- } \\
\text { ca: } 7 . \\
\text { Hemorragias post parto: } 3 \text {. } \\
\text { Parto prematuro: } 17 \text {. } \\
\text { Probable corioamnionitis o endo- } \\
\text { metritis: } 3 \text {. } \\
\text { Síndrome hipertensivo del emba- } \\
\text { razo: } 2 \\
\text { Promedio de edad gestacional al } \\
\text { parto: } 32 \text { semanas. }\end{array}$ & $\begin{array}{l}\text { RN COVID-19(+): } 1 \text { a las } 48 \mathrm{~h} \text {, } \\
\text { asintomático (no se identifica } \\
\text { aislamiento). } \\
\text { Ingreso a UCIN: } 21 \text {. } \\
\text { Apgar promedio } 7,9+-1.7 \text {. }\end{array}$ \\
\hline $\begin{array}{l}\text { Penfield, C., et } \\
\text { al. 2020. Nueva } \\
\text { York. USA. }{ }^{(16)}\end{array}$ & $\begin{array}{l}\text { Detection of severe } \\
\text { acute respiratory syn- } \\
\text { drome coronavirus } 2 \\
\text { in placental and fetal } \\
\text { membrane simples. }\end{array}$ & 32 parturientas. & $\begin{array}{l}\text { Hisopo de placenta de superficie } \\
\text { amniótica COVID-19 }(+): 1 . \\
\text { Frotis de membranas ovulares CO- } \\
\text { VID-19 }(+): 2 \text {. } \\
\text { Cesárea en enfermedad grave o } \\
\text { crítica: } 4 \text {. } \\
\text { Parto prematuro en enfermedad } \\
\text { grave o crítica: } 3 \text {. }\end{array}$ & $\begin{array}{l}\text { No se describen o no se encon- } \\
\text { traron alteraciones. }\end{array}$ \\
\hline
\end{tabular}




\begin{tabular}{|c|c|c|c|c|}
\hline $\begin{array}{l}\text { Autores, año } \\
\text { y ciudad }\end{array}$ & Título del estudio & Muestra & $\begin{array}{l}\text { Resultados obstétricos y } \\
\text { número de casos }\end{array}$ & $\begin{array}{l}\text { Resultados neonatales y } \\
\text { número de casos }\end{array}$ \\
\hline $\begin{array}{l}\text { Foong, K., Ban- } \\
\text { di, S., Bird, P. } \\
\text { W., \& Wei-Tze } \\
\text { Tang, J. } 2020 . \\
\text { Leicester, Reino } \\
{\text { Unido. }{ }^{(17)}}\end{array}$ & $\begin{array}{l}\text { COVID-19 in Neonates } \\
\text { and Infants: Progres- } \\
\text { sion and Recovery. }\end{array}$ & $\begin{array}{l}8 \text { lactantes CO- } \\
\text { VID-19(+) (Edad } \\
\text { entre } 5 \text { días a } 1 \\
\text { año). }\end{array}$ & $\begin{array}{l}\text { No se describen o no se encontra- } \\
\text { ron alteraciones. }\end{array}$ & $\begin{array}{l}\text { Neutropenia y trombocitosis: } 2 \text {. } \\
\text { Sospecha de sepsis: } 4 \text {. } \\
\text { Síntomas respiratorios: } 4 \text {. Un } \\
\text { prematuro no aislado COVID-19 } \\
\text { (+) a los } 5 \text { días requirió ingreso a } \\
\text { UCIP, tuvo hipotermia, apneas, } \\
\text { requirió VPP, fototerapia y tuvo } \\
\text { diagnóstico de CIA. }\end{array}$ \\
\hline $\begin{array}{l}\text { Vallejo, V., \& Ila- } \\
\text { gan, J. G. } 2020 . \\
\text { New York, USA. } \\
\text { (18) }\end{array}$ & $\begin{array}{l}\text { A Postpartum Death } \\
\text { Due to Coronavi- } \\
\text { rus Disease } 2019 \\
\text { (COVID-19) in the } \\
\text { United States }\end{array}$ & $\begin{array}{l}1 \text { gestante de } 37 \\
\text { semanas. }\end{array}$ & $\begin{array}{l}\text { Cesárea por condición materna. } \\
\text { Muerte materna por falla multior- } \\
\text { gánica. }\end{array}$ & $\begin{array}{l}\text { No se describen o no se encon- } \\
\text { traron alteraciones. }\end{array}$ \\
\hline $\begin{array}{l}\text { Blauvelt, C., et } \\
\text { al. 2020. San } \\
\text { Francisco, USA. } \\
\text { (19) }\end{array}$ & $\begin{array}{l}\text { Acute Respiratory } \\
\text { Distress Syndrome in } \\
\text { a Preterm Pregnant } \\
\text { Patient With Coro- } \\
\text { navirus Disease } 2019 \\
\text { (COVID-19). }\end{array}$ & $\begin{array}{l}1 \text { gestante de } 28 \\
\text { semanas. }\end{array}$ & $\begin{array}{l}\text { Cesárea x condición respiratoria } \\
\text { deteriorada. } \\
\text { Parto prematuro de } 28+6 \text { semanas. } \\
\text { Hemorragia postparto por atonía } \\
\text { uterina. } \\
\begin{array}{l}\text { Estudio placentario mostró co- } \\
\text { rioamnionitis aguda subclínica. }\end{array}\end{array}$ & $\begin{array}{l}\text { APGAR } 4 \text { y } 8 \text { al minuto y } 5 \text { mi- } \\
\text { nutos respectivamente. } \\
\text { Necesidad de reanimación neo- } \\
\text { natal por SDR. } \\
\text { Leucopenia. } \\
\text { Neutropenia. } \\
\text { Linfopenia, } \\
\text { Acidosis leve. }\end{array}$ \\
\hline $\begin{array}{l}\text { Martinelli, I., et } \\
\text { al. 2020. Milán, } \\
\text { Italia.(20) }\end{array}$ & $\begin{array}{l}\text { Pulmonary embo- } \\
\text { lism in a young preg- } \\
\text { nant woman with } \\
\text { COVID-19. }\end{array}$ & $\begin{array}{l}1 \text { gestante de } 29 \\
\text { semanas. }\end{array}$ & $\begin{array}{l}\text { Cesárea por condición materna } \\
\text { asociada al virus. }\end{array}$ & $\begin{array}{l}\text { Ingreso a UCIN con evolución } \\
\text { adecuada. }\end{array}$ \\
\hline $\begin{array}{l}\text { Kuusela, A., Na- } \\
\text { zir, M., Gimo- } \\
\text { vsky, M. } 2020 . \\
\text { Newark, USA. }{ }^{(21)}\end{array}$ & $\begin{array}{l}\text { Two cases of coro- } \\
\text { navirus 2019-related } \\
\text { cardiomyopathy in } \\
\text { pregnancy. }\end{array}$ & $\begin{array}{l}2 \text { gestantes con } \\
\text { infección grave } \\
\text { por Covid-19. } \\
\text { Caso } 1: 45 \text { años } \\
\text { G4P2 } 39+2 \text { sema- } \\
\text { nas. } \\
\text { Caso } \\
\text { G3P1 } 26 \text { años } \\
\text { nas. }\end{array}$ & $\begin{array}{l}\text { Preeclampsia: } 1 . \\
\text { Cesárea por condición materna } \\
\text { asociada al virus: } 1 . \\
\text { Cesárea preventiva por posible } \\
\text { agravación materna: } 1 \text {. }\end{array}$ & $\begin{array}{l}\text { No se describen o no se encon- } \\
\text { traron alteraciones. }\end{array}$ \\
\hline
\end{tabular}




\begin{tabular}{|c|c|c|c|c|}
\hline $\begin{array}{l}\text { Autores, año } \\
\text { y ciudad }\end{array}$ & Título del estudio & Muestra & $\begin{array}{l}\text { Resultados obstétricos y } \\
\text { número de casos }\end{array}$ & $\begin{array}{l}\text { Resultados neonatales y } \\
\text { número de casos }\end{array}$ \\
\hline $\begin{array}{l}\text { Xu, L., et al. } \\
\text { 2020, Wuhan, } \\
\text { China. }{ }^{(22)}\end{array}$ & $\begin{array}{l}\text { Clinical presenta- } \\
\text { tions and outcomes } \\
\text { of SARS-CoV-2 in- } \\
\text { fected pneumonia in } \\
\text { pregnant women and } \\
\text { health status of their } \\
\text { neonates. }\end{array}$ & $\begin{array}{l}5 \text { gestantes entre } \\
34+4 \text { y } 38+6 \text { sema- } \\
\text { nas con neumonía } \\
\text { leve. }\end{array}$ & $\begin{array}{l}\text { Cesárea por neumonía viral: } 3 \text {. } \\
\text { Parto prematuro por neumonía } \\
\text { materna: } 2 \text {. } \\
\text { Ligero desprendimiento prematu- } \\
\text { ro de placenta: } 1 \text {. } \\
\text { Polihidroamnios: } 1 \text {. } \\
\text { Placenta previa oclusiva: } 1 \text {. }\end{array}$ & $\begin{array}{l}\text { Erupciones cutáneas dispersas } \\
\text { en la cara y el cuerpo: } 1 . \\
\text { Todos los RN COVID-19(-). } \\
\text { RN pretérmino: } 2 \text {. } \\
\text { Bajo peso de nacimiento: } 1 \text {. }\end{array}$ \\
\hline
\end{tabular}

\footnotetext{
Patanè, L., et Vertical transmission of 22 gestantes con al. 2020. Ber- coronavirus disease 2019: 35 semanas de gamo, Italia. severe acute respiratory (23) 2 RNA on the fetal side of the placenta in pregnancies with coronavirus disease 2019-positive mothers and neonates at birth.
}

Estado fetal no tranquilizador: 1.

Cesárea por causa fetal: 1 .

Las 2 placentas de los RN infectados mostraron intervellositis crónica con presencia de macrófagos en espacio intervelloso y velloso.
RN COVID-19 (+): 2, a 1 RN se le permitió el alojamiento conjunto y la lactancia materna con máscara, el otro fue aislado inmediatamente postparto.

RN pretérmino: 1 .

Ingreso a UCIN: 1 .

Dificultad en la alimentación: ambos.

RN COVID-19 (+): 1 asintomático con aislamiento inmediato postparto. En los demás $\mathrm{RN}$, las

muestras de frotis de garganta, sangre, heces y orina dieron resultados negativos.
$\mathrm{Hu}, \mathrm{X}$, et al. Severe acute respi- 7 gestantes entre 2020. París, ratory syndrome $37+2$ y $41+2$ semaFrancia. ${ }^{(24)}$ coronavirus 2 (SARS-

CoV-2) vertical transmission in neonates born to mothers with coronavirus disease 2019 (COVID-19) pneumonia.
Cesáreas: 6.

Rotura prematura de membranas: 1.
Ingreso a UCIN: 3.

Pequeño para la edad gestacional: 1.

Bajo peso de nacimiento: varios (no especificado)
Cesárea por estad
ciado al virus: 9.

Cesárea por estado materno empeorado asociado al virus: 3 .

Preeclampsia: 2.

Restricción del crecimiento intrauterino: 1.

Parto prematuro: 5.

Hemorragia postparto: 1. 


\begin{tabular}{|c|c|c|c|c|}
\hline $\begin{array}{c}\text { Autores, año } \\
\text { y ciudad }\end{array}$ & Título del estudio & Muestra & $\begin{array}{c}\text { Resultados obstétricos y } \\
\text { número de casos }\end{array}$ & $\begin{array}{l}\text { Resultados neonatales y } \\
\text { número de casos }\end{array}$ \\
\hline $\begin{array}{l}\text { Alzamora, M. C., } \\
\text { Paredes, T., Cá- } \\
\text { ceres, D., Webb, } \\
\text { C. M., Valdez, L. } \\
\text { M., \& La Rosa, } \\
\text { M. 2020. } \\
\text { Nueva York, } \\
\text { USA. }{ }^{26)}\end{array}$ & $\begin{array}{l}\text { Severe COVID-19 } \\
\text { during pregnancy } \\
\text { and possible vertical } \\
\text { transmission. }\end{array}$ & $\begin{array}{l}\text { Gestante de } 33 \\
\text { semanas, } \\
\text { G3P2, } 41 \text { años }\end{array}$ & $\begin{array}{l}\text { Parto prematuro: } \\
\text { Cesárea por compromiso respira- } \\
\text { torio materno. }\end{array}$ & $\begin{array}{l}\text { RN COVID-19 }(+) \text { sin lactancia } \\
\text { materna, con aislamiento pos- } \\
\text { tparto inmediato. } \\
\text { Necesidad de soporte ventila- } \\
\text { torio por } 12 \text { horas luego venti- } \\
\text { lación a presión positiva. } \\
\text { Necesidad de ingreso a UCIN. }\end{array}$ \\
\hline $\begin{array}{l}\text { Khan, S., et al. } \\
2020 \text {. Zheng- } \\
\text { zhou, China. }{ }^{(27)}\end{array}$ & $\begin{array}{l}\text { Association of } \\
\text { COVID-19 with preg- } \\
\text { nancy outcomes in } \\
\text { health-care workers } \\
\text { and general women. }\end{array}$ & $\begin{array}{l}17 \text { gestantes de } \\
\text { entre } 35 \text { y } 41 \text { se- } \\
\text { manas. }\end{array}$ & $\begin{array}{l}\text { Parto prematuro: } 3 . \\
\text { Cesárea: } 17 . \\
\text { Rotura prematura de membranas: } \\
\text { algunas (no especifica). }\end{array}$ & 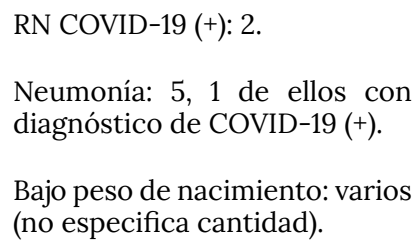 \\
\hline
\end{tabular}

Hang, Z. J., et al. Novel coronavirus in- $4 \mathrm{RN}$ de término

2020. Wuhan, fection in newborn con menos de 28 China. ${ }^{(28)} \quad$ babies aged $<28$ days in China. días de vida entre los 81.026 casos de COVID-19 al 13 de marzo de 2020.
Cesáreas: 4 (todas).

RN COVID-19 (+): 4, 2 por diagnóstico en hisopo nasofaríngeo y 2 por hisopo anal. (edad al diagnóstico entre 30 horas y 17 días). 3 de ellos fueron aislados al nacer. 3 tuvieron sintomatología de tipo respiratoria, fiebre y vómitos.

Días de hospitalización: entre 14 y 30

Chen, Y., et al. Infants born to moth- 4 gestantes.

Cesárea por preocupaciones de la Erupciones cutáneas: 2.

2020. Wuhan, ers with a new coro-

China. ${ }^{(29)}$ navirus (COVID-19).

Ulceraciones faciales: 2 .

Taquipnea neonatal: 1.

Ingreso a UCIN: 1 .

Zeng, L., et al. First case of neonate $1 \mathrm{RN}$ de térmi2020. Wuhan, with COVID-19 in Chi- no con 17 días de China. ${ }^{(30)}$ na.
No se describen o no se encontraron alteraciones. vida, hijo de padres COVID-19 $(+)$.
RN COVID-19(+) detectado en hisopado nasofaríngeo e hisopado anal.

Linfocitos CD4 y CD8 aumentados.

Neutropenia.

Alteraciones gastrointestinales.

Kamali M., Ja- Novel coronavirus in Neonato de 15 No se describen o no se enconfari, N., \& Eft- a 15-day-old neonate días de vida. ekhari, K. 2020. with clinical signs of Zanjan, Irán. ${ }^{(31)}$ sepsis, a case report.

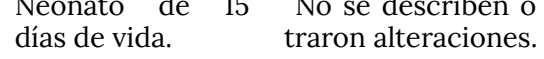

RN COVID-19(+) detectado en hisopado nasofaríngeo sin aislamiento.

Signos de sepsis.

Disnea.

Ingreso a UCIN. 


\begin{tabular}{|c|c|c|c|c|}
\hline $\begin{array}{l}\text { Autores, año } \\
\text { y ciudad }\end{array}$ & Título del estudio & Muestra & $\begin{array}{l}\text { Resultados obstétricos y } \\
\text { número de casos }\end{array}$ & $\begin{array}{l}\text { Resultados neonatales y } \\
\text { número de casos }\end{array}$ \\
\hline $\begin{array}{l}\text { Wang, Z., Wang, } \\
\text { Z., \& Xiong, G. } \\
2020 \text {, Wuhan, } \\
\text { China. }^{(32)}\end{array}$ & $\begin{array}{l}\text { Clinical character- } \\
\text { istics and laborato- } \\
\text { ry results of preg- } \\
\text { nant women with } \\
\text { COVID-19 in Wuhan, } \\
\text { China. }\end{array}$ & $\begin{array}{l}72 \text { mujeres de las } \\
\text { cuales } 30 \text { estaban } \\
\text { embarazadas. } \\
\text { EG promedio: } 38 \\
\text { semanas. }\end{array}$ & $\begin{array}{l}\text { Líquido amniótico meconial: } 4 \\
\text { Rotura prematura de membranas: } \\
6\end{array}$ & $\begin{array}{l}\text { No se describen o no se encon- } \\
\text { traron alteraciones. }\end{array}$ \\
\hline
\end{tabular}

\begin{tabular}{|c|c|c|c|c|}
\hline $\begin{array}{l}\text { Dong, L., et al. } \\
2020 \text {. Wuhan, } \\
\text { China. }{ }^{(33)}\end{array}$ & $\begin{array}{l}\text { Possible vertical } \\
\text { transmission of SARS- } \\
\text { CoV-2 from an infect- } \\
\text { ed mother to her new- } \\
\text { born. }\end{array}$ & $\begin{array}{l}\text { Primípara } \quad 34+2 \\
\text { semanas, } 29 \text { años. }\end{array}$ & $\begin{array}{l}\text { IgG e IgM para COVID-19 aumen- } \\
\text { tados. }\end{array}$ & $\begin{array}{l}\text { IgG e IgM elevadas } \\
\text { Citocina inflamatoria interleu- } \\
\text { kina- } 6 \text { aumentada. } \\
\text { Daño hepático. } \\
\text { Leucocitosis. } \\
\text { (RN con aislamiento inmediato) }\end{array}$ \\
\hline $\begin{array}{l}\text { Liu, D., et al. } \\
\text { 2020. Wuhan, } \\
\text { China. }^{(34)}\end{array}$ & $\begin{array}{l}\text { Pregnancy and perina- } \\
\text { tal outcomes of wom- } \\
\text { en with coronavirus } \\
\text { disease (COVID-19) } \\
\text { pneumonia: a prelim- } \\
\text { inary analysis. }\end{array}$ & $\begin{array}{l}15 \text { gestantes entre } \\
12 \text { y } 38 \text { semanas } \\
\text { de entre } 23 \text { y } 40 \\
\text { años. }\end{array}$ & $\begin{array}{l}\text { Cesáreas: } 10 \text {. } \\
\text { Parto prematuro por miedo a la } \\
\text { terapia antiviral: } 3 \text {. }\end{array}$ & $\begin{array}{l}\text { No se describen o no se encon- } \\
\text { traron alteraciones. }\end{array}$ \\
\hline $\begin{array}{l}\text { Chen, R., Zhang, } \\
\text { Y., Huang, L., } \\
\text { Cheng, B. H., } \\
\text { Xia, Z. Y., \& } \\
\text { Meng, Q. T. } \\
\text { 2020. Wuhan, } \\
\text { China. }\end{array}$ & $\begin{array}{l}\text { Safety and efficacy of } \\
\text { different anesthetic } \\
\text { regimens for partu- } \\
\text { rients with COVID-19 } \\
\text { undergoing Cesarean } \\
\text { delivery: a case series } \\
\text { of } 17 \text { patients. }\end{array}$ & 17 parturientas. & $\begin{array}{l}\text { Hipotensión significativa en anes- } \\
\text { tesia epidural continua: } 12 \text {, de un } \\
\text { total de } 14 \text { que la usaron. } \\
\text { Parto prematuro: } 3 \text {. }\end{array}$ & $\begin{array}{l}\text { No se describen o no se encon- } \\
\text { traron alteraciones. }\end{array}$ \\
\hline
\end{tabular}

Chen, S., et al. Pregnancy with new 2020. Wuhan, coronavirus infection: China. ${ }^{(36)}$ clinical characteristics and placental pathological analysis of three cases.
3 puérperas con Hemangioma coriónico: 1. diagnóstico de Covid-19(+) ante parto.
Infarto placentario multifocal: 1 .

Depósito de fibrina en o alrededor de vellosidades de placenta: 3 .

Parto prematuro: 1
RN pretérmino: 1 .

Bajo peso de nacimiento: 1. $\begin{array}{llllll}\text { Shanes, E. D., Placental pathology in } & \text { Muestra: } 16 \text { pla- } & \text { Al menos } 1 \text { signo de mala perfusión } & \text { No se describen o no se encon- } \\ \text { Mithal, L. } & \text { B., COVID-19 } & \text { centas de madres vascular materna: } 15 . & \text { traron alteraciones. }\end{array}$ Otero, S., Azad, Covid-19 $(+)$

H. A., Miller, E.

Muerte fetal intrauterina: 1.

S., \& Goldstein,

J. A. 2020, Chi-

cago, USA. ${ }^{(37)}$ 


\begin{tabular}{|c|c|c|c|c|}
\hline $\begin{array}{l}\text { Autores, año } \\
\text { y ciudad }\end{array}$ & Título del estudio & Muestra & $\begin{array}{l}\text { Resultados obstétricos y } \\
\text { número de casos }\end{array}$ & $\begin{array}{l}\text { Resultados neonatales y } \\
\text { número de casos }\end{array}$ \\
\hline $\begin{array}{l}\text { Prabhu et al., } \\
2020 \text {, New York } \\
\text { City } 38)\end{array}$ & $\begin{array}{l}\text { Pregnancy and post- } \\
\text { partum outcomes in } \\
\text { a universally tested } \\
\text { population for SARS- } \\
\text { CoV-2 in New York } \\
\text { City: a prospective co- } \\
\text { hort study. }\end{array}$ & $\begin{array}{l}675 \text { gestantes con } \\
\text { edad gestacional } \\
\text { promedio: } 39 \text { se- } \\
\text { manas. }\end{array}$ & $\begin{array}{l}\text { Cesáreas por causa fetal: } 4 . \\
\text { Cesáreas por causa obstétrica: } 11 . \\
\text { Hemorragia postparto: } 8 . \\
\text { Probables endometritis puerpera- } \\
\text { les: } 4 . \\
\text { Mala perfusión vascular fetal: } 14 . \\
\text { Mala perfusión vascular materna: } \\
8 . \\
\text { Líquido amniótico meconial: } 18 . \\
\text { Corioamnionitis histológica: } 1 . \\
\text { Vellitis:1. }\end{array}$ & $\begin{array}{l}\text { No se describen o no se encon- } \\
\text { traron alteraciones. }\end{array}$ \\
\hline
\end{tabular}

Ferraiolo et al., Report of Positive 1 gestante de 38 Estudio placentario positivo para No se describen o no se encon2020, Genova, Placental Swabs for semanas. COVID-19. traron alteraciones.

Italia. $^{(39)} \quad$ SARS-CoV-2 in an

Asymptomatic Pregnant Woman with COVID-19.

Nawsherwan, Impact of COVID-19 7

2020. Hubei, pneumonia on neona-

China. ${ }^{(40)} \quad$ tal birth outcomes. gestantes. Parto prematuro: 3.
Estado fetal no tranquilizador: $1 . \quad$ Bajo peso de nacimiento: 1.

Síndrome de distrés respiratorio: 3.

Ingreso a UCIN: 2.
Mendoza, et al. Pre-eclampsia-like 42 gestante de Preeclampsia: 5.

2020. Barcelo- syndrome induced by promedio 32 sena, España. ${ }^{(41)}$ prospective observational study.

No se describen o no se encontraron alteraciones.
Futterman, I., COVID-19 and HELLP: 2 gestantes, de 22 Muerte fetal intrauterina: 1. Toaff, M., Navi, Overlapping Clinical y 29 semanas. L., \& Clare, C. A., Pictures in Two Grav2020, New York, id Patients USA. ${ }^{(42)}$
Estado fetal no tranquilizador:1.

Depósito de fibrina perivelloso: 2.

Infarto placentario: 1 .

Intervellositis: 1.

Leche de madre 1: COVID 19 (-).

Leche de madre 2: COVID-19 por 4 días consecutivos.
RN COVID-19 (+): 1 (no identifica aislamiento inmediato postparto).

Ingreso a UCIN: 1.

Leucocitosis: 1.

Alteración de la función hepática y renal: 1 .

RN 1: COVID-19 (-)

2020 Alemania. CoV-2 in human breast milk.
(+) RN 2: COVID-19(+) (Ningún RN tuvo aislamiento inmediato postparto) 